\title{
Relationship of energy charge and toxin content of Microcystis aeruginosa in nitrogen-limited or phosphorous-limited cultures
}

\author{
Ruihua Dai ${ }^{\mathrm{a}, \mathrm{b}}$, Huijuan $\mathrm{Liu}^{\mathrm{a}}$, Jiuhui Qu ${ }^{\mathrm{a}, *}, \mathrm{Xu}_{\mathrm{Zhao}}{ }^{\mathrm{a}}, \mathrm{Jia} \mathrm{Ru}^{\mathrm{a}}$, Yining Hou ${ }^{\mathrm{a}, \mathrm{b}}$ \\ ${ }^{a}$ State Key Laboratory of Environmental Aquatic Chemistry, Research Center for Eco-Environmental Sciences, \\ Chinese Academy of Sciences, Beijing 100085, China \\ ${ }^{\mathrm{b}}$ Graduate School of the Chinese Academy of Sciences, Beijing 100039, China
}

Received 21 September 2007; received in revised form 25 November 2007; accepted 29 November 2007

Available online 3 December 2007

\begin{abstract}
Effects of nitrogen-limitation and phosphorus-limitation on microcystin (MC) content and energy charge (EC) of the Microcystis aeruginosa were investigated in batch cultures and semi-continuous cultures. In batch cultures, nitrogenlimitation retarded the MC synthesis and phosphorus-limitation had little effects on MC production. The EC remained constant in nitrogen-limited cultures, while it decreased largely when phosphorus was extinct in phosphorus-limited culture. In the semi-continuous cultures, MC production in nitrogen- and phosphorus-limited cultures increased with the increase of dilution rate; however, MC content in phosphorus-limited cultures was more than that in nitrogen-limited cultures. The EC in nitrogen-limited cultures remained constant and in phosphorus-limited cultures increased with the increase of dilution rate. In phosphorus-limited semi-continuous cultures, a direct relationship between EC and MC content was demonstrated. No correlation was observed in nitrogen-limited semi-continuous cultures. Based on the above analysis, a mechanism of nitrogen and phosphorus effect on the MC synthesis was suggested, that the MC synthesis was determined by the combination of necessary enzymes and precursors and EC.
\end{abstract}

(C) 2007 Elsevier Ltd. All rights reserved.

Keywords: Energy charge; Microcystin; Nitrogen-limited; Phosphorus-limited; Microcystis aeruginosa

\section{Introduction}

Cyanobacteria like Microcystis, Oscillatoria and Nodularia are able to form massive blooms frequently in eutrophic lakes and polluted water (Elke and Claudia, 2006; Rinta-Kanto et al., 2005). Among the toxic cyanobacteria, Microcystis aeruginosa is

\footnotetext{
Ethical statement: This paper is new. The work described has not been submitted elsewhere for publication, in whole or in part, and all the authors listed have approved the manuscript.

*Corresponding author. Tel.: +861062849151; fax: + 861062923558 .

E-mail address: jhqu@rcees.ac.cn (J. Qu).
}

commonly observed in highly eutrophic lakes (Shi et al., 2005; Watanabe and Oishi, 1985). The microcystins (MCs) produced by $M$. aeruginosa have been the cause of several poisonings of domestic animals and wildlife around the world, and they also pose a health hazard for humans through the use of water for drinking and recreation (Elke and Claudia, 2006; Hoeger et al., 2007).

Many environmental factors influence the production of MC and remain extensively studied. The effects of nitrogen (Downing et al., 2005), phosphorous (Oh et al., 2000; Wicks and Thiel, 1990), N:P ratio (Lee et al., 2000), light intensity 
and quality (Utkilen and Gjalme, 1992), temperature (Maria and Daniel, 2005) and pH (Jahnichen et al., 2001) have been investigated to various degrees in both batch and continuous cultures. These environmental factors influencing MC production, either directly or indirectly via regulating mechanisms, should further be identified and studied extensively so that this knowledge can be applied to prevent, control or at least understand naturally occurring $M$. aeruginosa blooms. Among these factors, nitrogen and phosphorus are crucial because they are important nutrients for cyanobacterial growth, and especially nitrogen, which is a key element in MCs. Therefore, many scientists have focused on the effects of nitrogen and phosphorus on MC production using the Microcystis species (Downing et al., 2005; Lee et al., 2000; Song et al., 1998; Vezie et al., 2002). However, they mainly discussed the effects of nitrogen and phosphorus on MC production, while the mechanisms of nitrogen and phosphorus effects on MC content and production were not furthermore studied.

Bickel et al. (2000) showed that adenylate energy charge (EC) indirectly affected the production of MCs and indicated that EC and MC production had a positive correlation in phosphorus- and irondepletion cultures. However, they did not discuss the relationship between nitrogen and EC, and also did not explain how nitrogen and phosphorus affected MC production.

It is well known that the adenine nucleotides, including adenosine triphosphate (ATP), adenosine diphosphate (ADP) and adenosine monophosphate (AMP), are ubiquitous in living organisms. ATP, ADP and AMP stoichiometrically couple all of the metabolic sequences of a living cell, and are responsible for coupling intracellular energy-producing and energy-requiring metabolic reactions. The regulation of enzyme function and the control of biosynthetic processes have resulted in the formulation of the adenylate EC concept. The amount of metabolically available energy that is momentarily stored in the adenylate system is linearly related to the mole fraction of ATP plus half the mole fraction of ADP. This parameter referred to the EC of the adenylate pool (Atkinson and Walton, 1967). Chapman et al. (1971) demonstrated the EC, which was the fractional degree to which the AMPADP-ATP system was filled with high-energy phosphates. As defined by Atkinson and his colleagues (Atkinson and Walton, 1967; Chapman et al., 1971), the adenylate EC was equal to one-half of the number of anhydride-bound phosphate groups per adenine moiety,

$\mathrm{EC}=\frac{[\mathrm{ATP}]+1 / 2[\mathrm{ADP}]}{[\mathrm{ATP}]+[\mathrm{ADP}]+[\mathrm{AMP}]}$,

and was therefore the amount of metabolic energy stored in the adenine nucleotide pool.

Effects of nitrogen and phosphorus on the production of cyclic peptide hepatotoxins in cyanobacteria have been investigated for nearly 50 years. Numerous studies focused on the correlation between toxin production and concentrations of nitrogen or phosphorus. Few studies discussed the relation between the physiological state (in addition to cell cycle) and MC production. This study discussed the correlation between the EC (energy state) and the cellular content of MC of $M$. aeruginosa in nitrogenlimited and phosphorus-limited batch cultures and semi-continuous cultures. The positive correlation between $\mathrm{EC}$ and $\mathrm{MC}$ content in phosphorus-limited semi-continuous cultures was observed and no relationship between EC and MC content was found in nitrogen-limited cultures, and the effect mechanisms of nitrogen and phosphorus on MC production were proposed.

\section{Materials and methods}

\subsection{Microcystis aeruginosa and cultures}

An axenic strain of $M$. aeruginosa, which was isolated from Dianchi Lake in China, was obtained from the Institute of Hydrobiology, Chinese Academy of Science.

M. aeruginosa was maintained in BG11 (Rippka et al., 1979) medium. Initial $\mathrm{pH}$ of the medium was adjusted to 8.5 using $1.0 \mathrm{~mol} / 1$ hydrochloric acid and sodium hydroxide solutions. The medium and all experimental utensils were kept at $121^{\circ} \mathrm{C}$ for $20 \mathrm{~min}$ in order to sterilize the bacteria. Cultures in exponential phase were concentrated by centrifugation and washed three times with sterile distilled water and then inoculated in the same growth medium, but without nitrogen and phosphorus source for a week to exhaust the nitrogen and phosphorus in the cells. $M$. aeruginosa were then added to a series of 2.51 flat-bottomed bottles containing 21 medium.

All samples were run in triplicate bottles at $26^{\circ} \mathrm{C}$ and a photon flux of $8-12 \mu \mathrm{w} / \mathrm{cm}^{2}$ with a $12 \mathrm{~h}$ photoperiod. All flat-bottomed bottles were shaken 
by hand three times every day during the maintenance and experimental stages.

\subsection{Experimental design}

The batch-culture experiments were performed in nitrogen- and phosphorus-sufficient (control) culture, nitrogen-deficient culture and phosphorusdeficient culture. The concentrations of nitrogen and phosphorus in all cultures are listed in Table 1. $M$. aeruginosa, whose nitrogen and phosphorus were exhausted, already were fed with 21 BG11 medium in 2.51 flat-bottomed bottles. After inoculation, at given times during cell growth and at 2-day intervals, samples were aseptically removed for further analysis. Cell number, MC content and EC values were analyzed by the following methods. Nitrate was determined by a U-3010 spectrophotometer (Hitachi, Japan) and phosphate was assayed by the molybdenum-antimony anti-spectrophotometric method, which were described in monitoring and determination methods for water and wastewater (EPC, 2002).

In semi-continuous culture experiments, the concentrations of nitrogen and phosphorus are shown in Table 1. Each culture was inoculated with $50 \mathrm{ml}$ of exponentially growing, axenic $M$. aeruginosa cells, which were without nitrogen and phosphorus medium for 1 week. Each 2.51 flat-bottomed bottle contained 21 BG11 medium. After the culture reached logarithmic growth, the inflow of media was adjusted to give dilution rates of $0.05,0.1$, $0.15,0.25$ and $0.35 \mathrm{day}^{-1}$ at 2-day intervals. After 20 days, the optical density at $680 \mathrm{~nm}$ of the semi-continuous culture was measured at intervals of 2 days with the U-3010 spectrophotometer (Hitachi, Japan). Once the culture reached a constant optical density and cell density remained steady for a minimum of 2 weeks, the culture was harvested. Then the cell number, MC and ATP, ADP and AMP contents were measured for 5 successive days.

\subsection{Measurement of cell number}

After straining with Lugol's iodine solution, the population of $M$. aeruginosa cells was counted under a microscope using a hemocytometer to calculate the density of cyanobacterial cells. The precision of cell counts was determined to be less than $20 \%$.

\subsection{Determination of $M C$ within the cells}

Fifty milliliters of cyanobacterial culture solution was taken at predetermined intervals. The samples were centrifuged at $8000 \mathrm{r} / \mathrm{min}$ for $10 \mathrm{~min}$ and then the supernatant was discarded. The cell pellet was frozen under $-20{ }^{\circ} \mathrm{C}$ before further analysis. From the freeze-dried cell material, $20 \mathrm{ml} \mathrm{50 \%} \mathrm{methanol-}$ water solution was added to the cell pellet to extract MC within cyanobacterial cells. The extraction solution was staved using an Ultrasonic Cell Disruptor (JY92-2D, SCIENTZ, China) for $30 \mathrm{~min}$. The solution was centrifuged at $10,000 \mathrm{r} / \mathrm{min}$ for $20 \mathrm{~min}$ and then the supernatant was applied to a C-18 cartridge. The cartridge containing the MC was rinsed with $10 \mathrm{ml}$ water. The MCs were finally eluted from the C-18 cartridge with $10 \mathrm{ml}$ methanol. The eluant was evaporated by mild nitrogen gas. Finally, the residue was dissolved in $1 \mathrm{ml}$ distilled water that was measured for MC. The MC content in the cells was calculated from the ratio of the concentration of $\mathrm{MC}$ in the extraction solution $(\mathrm{mg} / \mathrm{l})$ to the cells in the subsamples (cells $/ \mathrm{ml})$. The data presented here were the average values of three parallel samples with the standard deviation.

Standard MC-RR and MC-LR were bought from Sigma Co. (USA, 98\% purity) and samples of MC-RR and MC-LR were all analyzed by a reversedphase high-performance liquid chromatograph (HPLC, Hitachi L-2000) with a reverse ZORBAX SB-C18 column $(4.6 \mathrm{~mm} \times 250 \mathrm{~mm}$, Agilent Co., USA) and a diode array detector at $238 \mathrm{~nm}$. The mobile phase was $35 \%(\mathrm{v} / \mathrm{v})$ acetonitrile-water

Table 1

Concentrations of nitrogen and phosphorus in batch cultures and semi-continuous cultures

\begin{tabular}{|c|c|c|c|c|c|c|}
\hline \multirow[t]{2}{*}{ Concentration $(\mu \mathrm{mol} / \mathrm{l})$} & \multicolumn{3}{|c|}{ Semi-continuous cultures } & \multicolumn{3}{|c|}{ Batch cultures } \\
\hline & Control & N-limited & P-limited & P-limited & N-limited & Control \\
\hline Nitrogen & $1790^{\mathrm{a}}$ & 50 & $1790^{\mathrm{a}}$ & 895 & 119 & 895 \\
\hline Phosphorous & $175^{\mathrm{a}}$ & $175^{\mathrm{a}}$ & 1 & 11.7 & 35 & 35 \\
\hline
\end{tabular}

\footnotetext{
${ }^{\mathrm{a}}$ The concentration of BG11 medium
} 
solution containing $0.05 \%(\mathrm{v} / \mathrm{v})$ of frozen acetic acid. The flow rate was $1.0 \mathrm{ml} / \mathrm{min}$ and the injection amount was $20 \mu \mathrm{l}$. The MCs were identified by their UV spectra and retention times, and by spiking the sample with a purified standard of MC (Sigma).

\subsection{Extraction and analysis of the adenine nucleotides}

The adenine nucleotides were extracted by $2 \mathrm{mmol} / 1 \mathrm{MgSO}_{4}$ solution and ultrasonic treatment. Ten milliliters of culture was centrifuged at $8000 \mathrm{r} / \mathrm{min}$ for $10 \mathrm{~min}$ and the supernatant was spilled. Then $1 \mathrm{ml} 90^{\circ} \mathrm{C}$ solution of $\mathrm{MgSO}_{4}$ was rapidly injected into the centrifugal tube. The solution was kept in boiling water for $10 \mathrm{~min}$ and was staved by ultrasonic for $10 \mathrm{~min}$ and then was centrifuged. The supernatant was transferred to $1 \mathrm{ml}$ plastic vials, which was frozen for future analysis or analyzed directly. There was no significant difference between samples frozen for up to $1 \frac{1}{2}$ months and samples analyzed immediately.

As measures for the amount of EC in the cells including ATP, ADP and AMP, their contents were determined by a reversed-phase high-performance liquid chromatograph (Hitachi-L2000). The separation was performed on an ODS $(4.6 \mathrm{~mm} \times 250 \mathrm{~mm}$, Allsphere) reverse-phase column and the mobile phase was a methanol- $0.05 \mathrm{~mol} / 1$ phosphate buffer ( $\mathrm{pH}=6.0$, containing $1 \mathrm{mmol} / 1$ EDTA) whose ratio was $2.5 \%: 97.5 \%(\mathrm{v} / \mathrm{v})$. The detector was set at $259 \mathrm{~nm}$. The flow rate was $1 \mathrm{ml} / \mathrm{min}$. Their content was quantified by calibrating against ATP, ADP and AMP standards (Sigma).

\section{Results and discussions}

\subsection{Analysis of $M C-R R$ and $M C-L R$}

Fig. 1(a) shows the HPLC profile of standard solutions of $5 \mathrm{mg} / \mathrm{l}$ of MC-RR and MC-LR. Peaks of MC-RR and MC-LR appeared at 4.33 and $6.76 \mathrm{~min}$, respectively. The wavelength of $238 \mathrm{~nm}$ was the maximum absorbance of MC-RR and MC-LR, which agreed with the literature (Lawton et al., 1994; Moollan et al., 1996) and was used for the detection of MC on HPLC in this experiment. Good linear relationships between peak areas and the standard concentrations of MC-RR and MC-LR were obtained at the wavelength of $238 \mathrm{~nm}$ $\left(R^{2}=0.998\right.$ and 0.999). Fig. 1(b) was the HPLC profile of MC in the cells of $M$. aeruginosa. It was obvious that the MC in cells was MC-LR; that is, $M$. aeruginosa only produced MC-LR but no MC-RR.

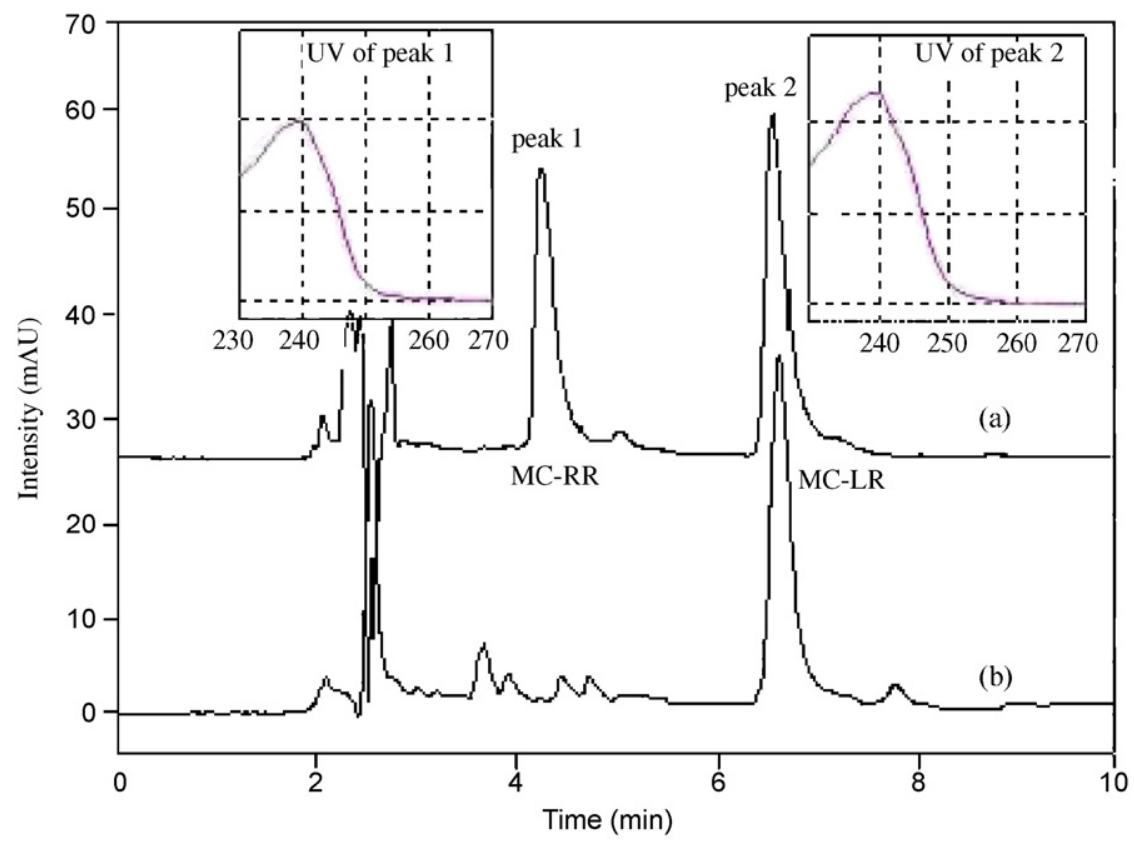

Fig. 1. HPLC profiles for (a) standard MC-RR $(\mathrm{RT}=4.33 \mathrm{~min})$ and MC-LR $(\mathrm{RT}=6.76 \mathrm{~min})$ and (b) profiles for MC-LR in the cells of Microcystis aeruginosa. Concentrations of standard MC-RR and LR are $5 \mathrm{mg} / \mathrm{l}$ each. 


\subsection{Analysis of ATP, ADP and AMP}

Fig. 2 shows the HPLC profile of standard solutions of $5 \times 10^{-8} \mathrm{~mol} / \mathrm{l}$ of ATP, ADP and AMP. It is obvious that peaks of ATP, ADP and AMP were separated clearly and appeared at 3.627, 4.347 and $7.200 \mathrm{~min}$, respectively. Good linear relationships between peak areas and the standard concentrations of ATP, ADP and AMP were obtained at the wavelength of $259 \mathrm{~nm}\left(R^{2}=0.997\right.$, 0.997 and 0.999). All of these results showed that HPLC (Hitachi-L2000) could be utilized to detect ATP, ADP and AMP and to quantify the contents of ATP, ADP and AMP in this experiment. The value of EC was determined by Eq. (1) via concentrations of ATP, ADP and ATP.

\subsection{Microcystin and energy charge in batch culture}

Batch culture is a suspension culture in which cells grow in a finite volume of liquid nutrient medium and follow a sigmoid pattern of growth. It is highly dynamic and nutrient supply becomes less and less with incubation time prolonged. Fig. 3 shows the variations of MC contents, EC and biovolume in batch cultures with different conditions. Fig. 3(a) was achieved under the condition that nitrogen and phosphorus were abundant (control), and it indicated that the growth of $M$. aeruginosa was flourishing and the content of
MC-LR within the $M$. aeruginosa cells decreased in the lag and late-exponential growth phase but peaked in the early or mid-exponential phase on the 9th day. The EC in Fig. 3(a) was constant and remained at about 0.8 in all growth periods. As shown in Fig. 3(b), phosphorus was deficient and was exhausted on the 14th day, but nitrogen was present all the time in the batch culture. Fig. 3(b) indicated the growth and MC content in phosphorus-limited culture, which were similar to that of control (Fig. 3(a)). However, it was different when the EC suddenly declined when phosphorus was exhausted on the 14th day. As shown in Fig. 3(c), nitrogen was deficient and was exhausted on the 9th day. The growth of $M$. aeruginosa was retarded by scarce nitrogen and was stationary on the 9th day. Therefore, the biomass in this culture was far lower than that of the control (Fig. 3(a)). Moreover, MC production was also lower than that of the control (Fig. 3(a)) and phosphorus-limited culture (Fig. 3(b)), and peaked on the 7th day. During the cell growth cycle, the EC had a value of about 0.8 .

In batch cultures, all cultures had a characteristic convex pattern of MC content with time as shown in Fig. 3, rising to a peak in mid-exponential growth and decreasing rapidly during the plateau phase. Not only our data, but also the results of Kameyama et al. (2004), Lee et al. (2000) and Song et al. (1998) showed a similar mode. A little

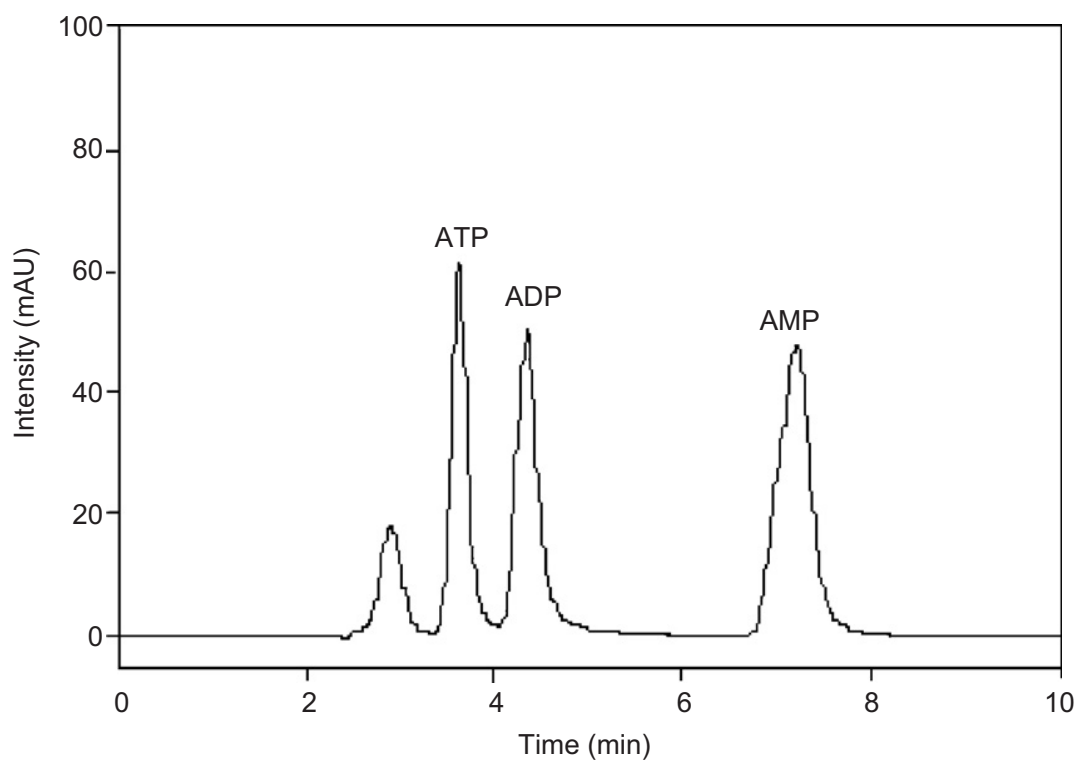

Fig. 2. HPLC profiles for standard ATP $(\mathrm{RT}=3.627 \mathrm{~min})$, ADP $(\mathrm{RT}=4.347 \mathrm{~min})$ and $\mathrm{AMP}(\mathrm{RT}=7.200 \mathrm{~min})$. Concentration of standard ATP, ADP and AMP are $5 \times 10^{-8} \mathrm{~mol} / \mathrm{ml}$ each. 
a

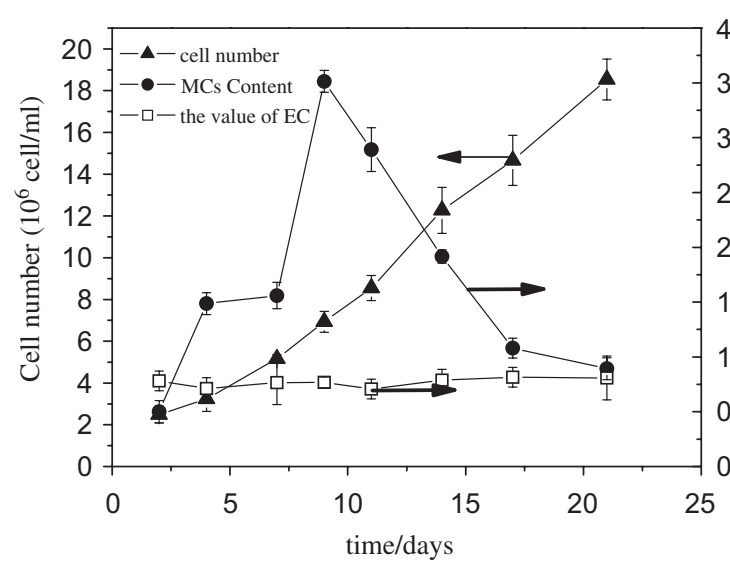

b

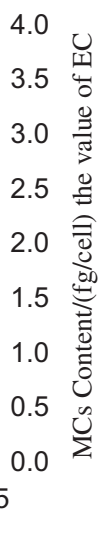

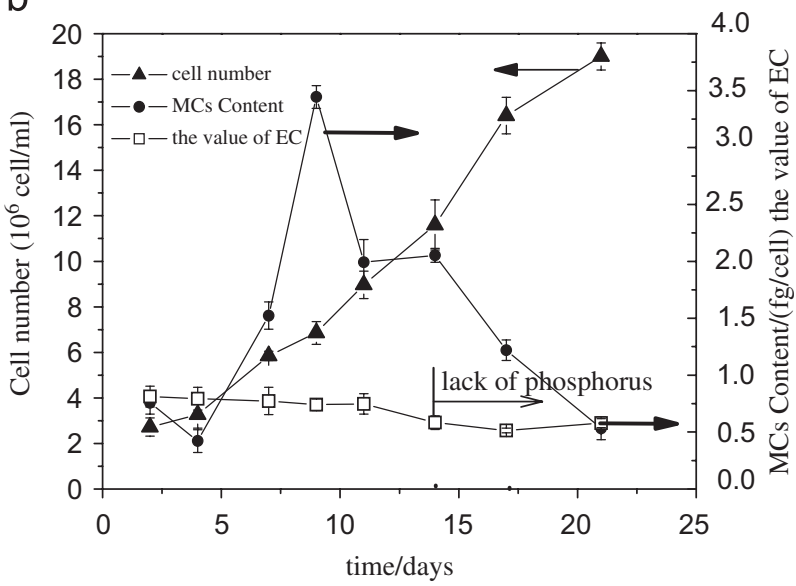

C

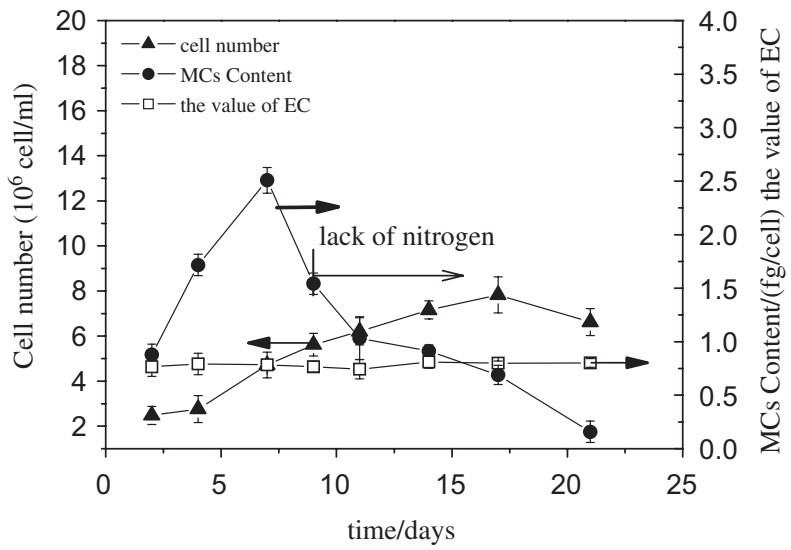

Fig. 3. Microcystin contents and energy charge in batch cultures. (a) Control, (b) phosphorus deficient, (c) nitrogen deficient.

difference was in growth and MC content between the control (Fig. 3(a)) and phosphorus-deficient culture (Fig. 3(b)); however, the large difference was in growth and MC content between the control (Fig. 3(a)) and nitrogen-deficient culture (Fig. 3(c)). This phenomenon testified that $M$. aeruginosa needed less phosphorus than nitrogen to support their growth and could produce almost equal MC in phosphorus-limited cultures, and also demonstrated that the nitrogen source was very important for MC synthesis.

With respect to EC, its value remainded at about 0.8 in nitrogen-limited culture and nitrogenabundant culture (control), while it decreased at the time when phosphorus was exhausted. Thus phosphorus had a large effect on EC.

Due to the variation of nutrient concentration, biomass and stage of physiology in batch cultures, we could not observe the relationship between EC and MC content. Thus, the following experiments were performed to reveal the relationship between $\mathrm{EC}$ and $\mathrm{MC}$ production.

\subsection{Microcystin content, energy charge and their correlation in semi-continuous cultures}

In order to obtain the relevancy between EC and MC production, EC and MC production were studied in semi-continuous cultures. Batch and semi-continuous cultures differ in that in a continuous culture system, nutrients are supplied to the cell culture at a constant rate, and in order to maintain a constant volume, an equal volume of cell culture is removed. This allows the cell population to reach a "steady state" (e.g. the growth rate and the total number of cells per milliliter of culture remain constant).

Fig. 4 shows the variations of $\mathrm{MC}$ content and EC value with increase of dilution rate in control (nitrogen and phosphorus were abundant), 
a

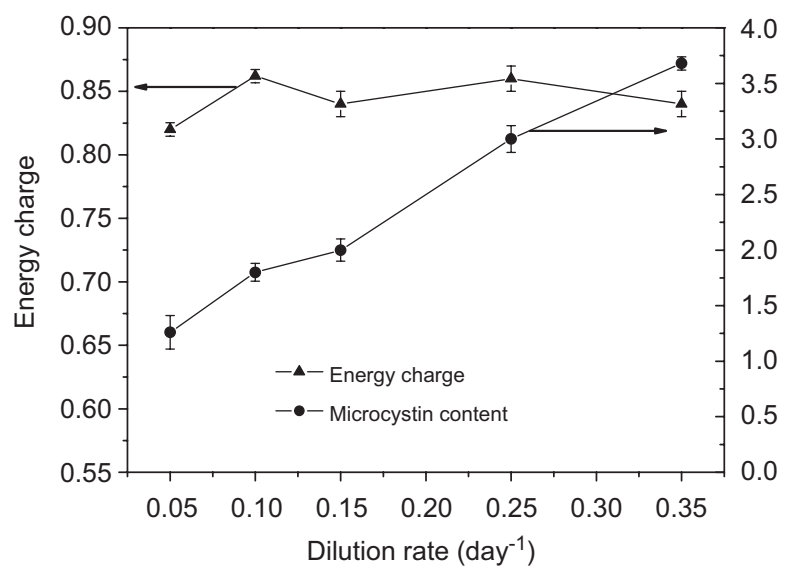

b

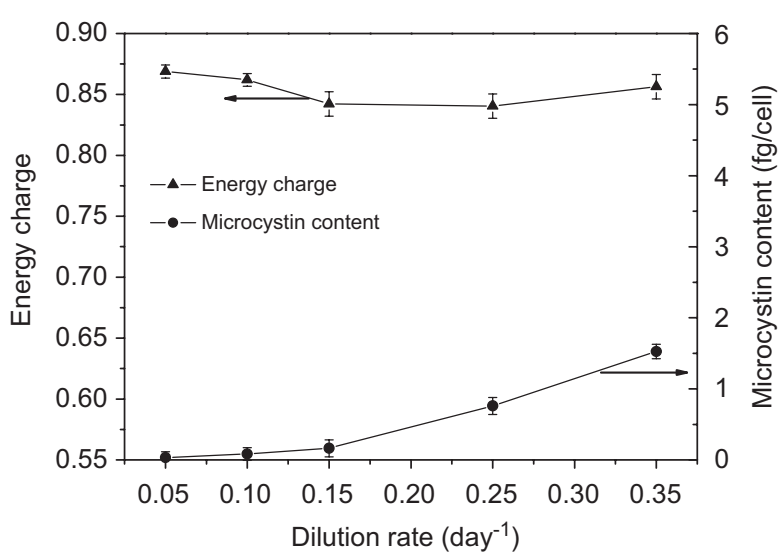

C

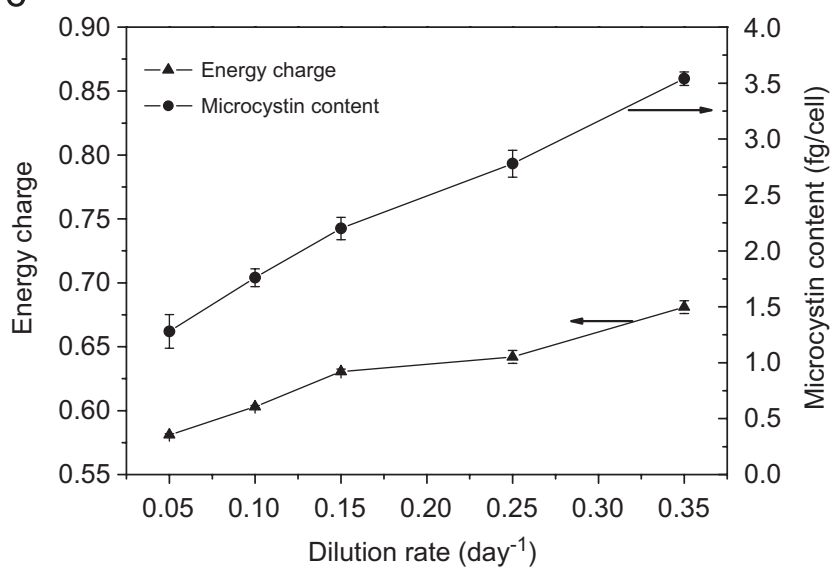

Fig. 4. Microcystin contents and energy charge in control (a), nitrogen-limited semi-continuous culture (b) and phosphorus-limited semicontinuous culture (c).

nitrogen-limited and phosphorus-limited semicontinuous cultures. In the control culture (Fig. 4(a)), the MC content varied from 1.26 to $3.68 \mathrm{fg} /$ cell and EC from 0.82 to 0.86 . It was obvious that $\mathrm{MC}$ content increased and the EC changed little with the increase of dilution rate. In the nitrogen-limited culture, the MC content varied from 0.03 to $1.52 \mathrm{fg} / \mathrm{cell}$ and the EC value from 0.84 to 0.87 . With the increase of dilution rate, the $\mathrm{MC}$ content also increased, while the EC value was almost constant as shown in Fig. 4(b). In the phosphorus-limited culture, the MC content varied from 1.76 to $3.57 \mathrm{fg} / \mathrm{cell}$. The EC value ranged from 0.58 to 0.68 . Fig. 4(c) shows that the MC content increased and the EC value also increased with the increase of dilution rate. Maximal analytical error of MC content and EC value during constant optical density was determined to be less than $20 \%$.
Comparing Fig. 4(a) with Fig. 4(b) and Fig. 4(c), it was obvious that MC content in nitrogen-limited culture was lower than that of control cultures, while the trend of EC was similar with that of the control culture. In phosphorus-limited cultures, the MC content was similar to that of the control culture; however, EC was lower than that of the control culture. As shown in Fig. 4(b) and (c), cells adapted to a very low supply of phosphorus in the semi-continuous culture had higher toxin content than that of the nitrogen-limited culture although their MC content both increased with the increase of dilution rate. With increase of dilution rate, the EC value in nitrogen-limited and phosphoruslimited cultures was largely different. The former was almost constant while the latter increased. This phenomenon could testify that phosphorus had a large effect on EC and nitrogen had little effect on 
it. These results were consistent with the results and phenomena in batch cultures.

The optical density of semi-continuous cultures reflected approximately steady-state physiological and nutritional conditions (details are not listed). At different dilution rates, $M$. aeruginosa were all at a constant stage and had a stable physiological state. In such a stage, the effect of nitrogen and phosphorus on MC production could be really reflected. They thus provided useful comparisons with nutrient limitation effects on MC production in the low nitrogen and low phosphorus batch cultures. In batch cultures, as shown in Fig. 3(a) and (c), whereas nitrogen was abundant, scarce or even extinct, the EC value was approximately 0.8 and was almost constant. When phosphorus was absent in the medium, the EC value just fell to below 0.6. Based on the above results, we can only judge that phosphorus affected the EC value and nitrogen had no effect on it, and did not infer the relation between EC and MC content because batch culture was varied and changed rapidly with incubation time. The nutrient supply and environment were invariant in semi-continuous culture. MC production in the semi-continuous cultures reflected balanced growth where the toxin biosynthetic machinery and the necessary enzymes were in equilibrium. Therefore, changes of $\mathrm{EC}$ and $\mathrm{MC}$ production could be reflected distinctly in these cultures.

Fig. 5 shows the correlations between MC content and EC in nitrogen-limited and phosphorus-limited semi-continuous cultures. Fig. 5(a) shows that there was no correlation between MC content and EC in nitrogen-limited semi-continuous cultures. However, in the phosphorous experiment, a positive correlation was observed between EC and MC content as shown in Fig. 5(b). The correlation coefficient was 0.983 for the phosphorous experiment. The significance of the correlation was given at $P \leqslant 0.01$. This partly agreed with the study of Bickel and Lyck (2000), which believed that a positive relationship between $\mathrm{EC}$ and $\mathrm{MC}$ content was in phosphorus and iron continuous culture.

\subsection{The regulating mechanism of nitrogen and phosphorus on microcystin production}

In the low nitrogen culture (Figs. 3(c) and 4(a)), the shortage of nitrogen resulted in low toxin content and growth. In batch cultures, compared with the control, MC content in nitrogen-limited cultures was less than that of $30 \%$ at the peak value. Especially after nitrogen was exhausted, MC content decreased quickly and was only $0.15 \mathrm{fg} /$ cell on the 22nd day. Results from Boyer et al. (1987) suggested that if our batch culture experiment had been extended, MC content might have decreased even further. This was not surprising, since nitrogen was a key element participating in the process of MC synthesis and as a necessary raw material was synthesized into the MC. Furthermore, nitrogen accounts for over $14 \%$ in the molecular weight of a

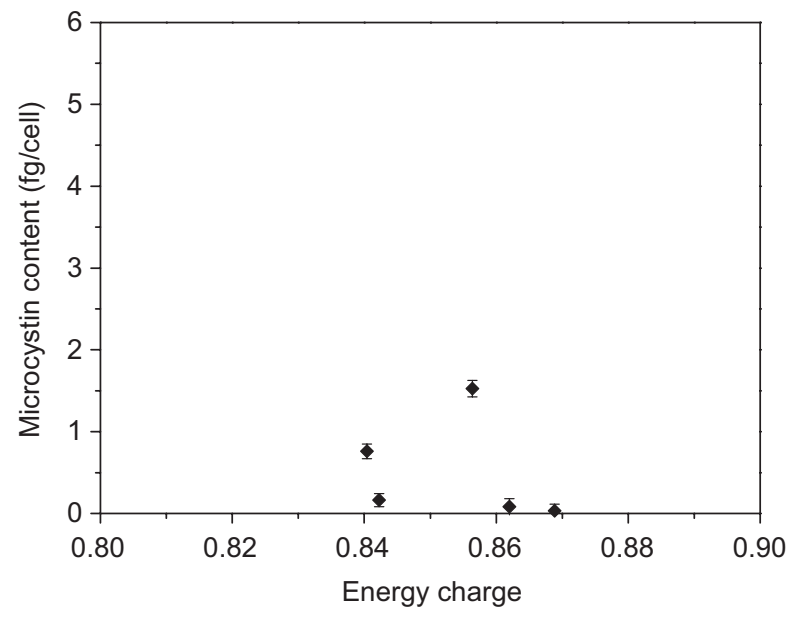

b

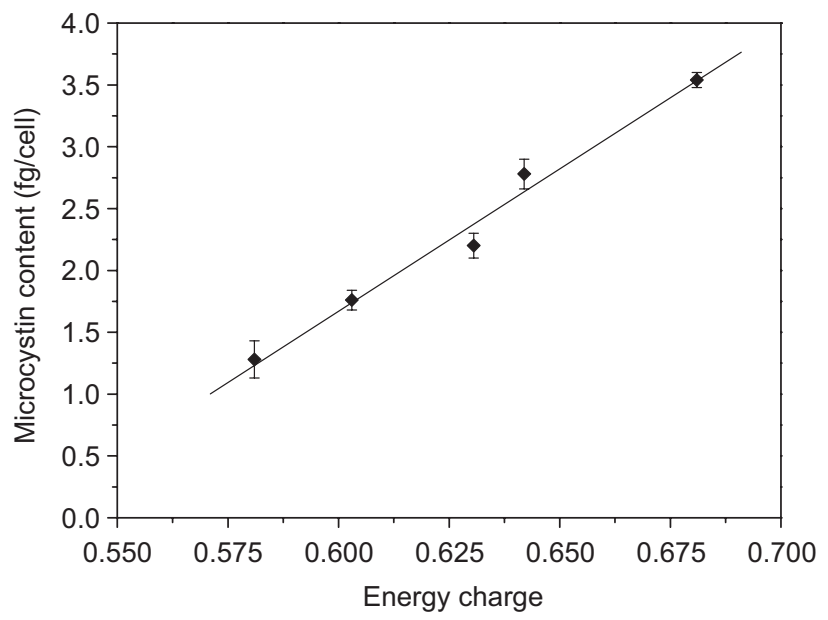

Fig. 5. Correlation between microcystin contents and energy charge in nitrogen-limited semi-continuous cultures (a) and phosphoruslimited semi-continuous cultures (b). 
MC-LR (Botes et al., 1985). The reason that nitrogen-limitation resulted in low MC content was that the synthesis process was obstructed by the exiguous nitrogen from the headstream because of absent raw material. The above results demonstrated that nitrogen was important and its concentration directly influenced the MC synthesis. The two nitrogen-limited modes (Figs. 3(c) and 4(a)) could confirm these results.

Phosphorus was not a component in the MC molecule; therefore, it did not directly attend the process of $\mathrm{MC}$ synthesis. But phosphorus was important in trapping, conserving, transporting and donating energy by the so-called high-energy phosphate bond $(\sim \mathrm{P})$ in the metabolism (Hegeman, 1969 ). During the process of metabolism, phosphorus was assimilated to synthesize the nucleotides (ATP and ADP) to offer the energy for theMC synthesis, and thus regulated enzyme function and controlled the pathway of MC synthesis. It was reported that phosphorus was incorporated into organic high-energy compounds (ATP) by the process of substrate phosphorylations in the cell of $M$. aeruginosa in this condition (Cheng and Mao, 1987). During the process of substrate phosphorylations, there was a stoichiometric coupling agent between phosphorus and the adenine nucleotides. In a phosphate-limited culture, the EC could fall below 0.7 or even lower due to lack of stoichiometry between the adenylate pool and inorganic orthophosphate. Phosphate starvation had been implicated in decreased ATP pool sizes in the cells (Benzion, 1977; Sakshaug and Holm-Hansen, 1977). Cells in semi-continuous culture that successfully adapted to the new conditions changed their energetic status and contained a higher or lower ratio of $\mathrm{MC}$, depending on the energetic status at that moment.

In conclusion, these results indicated that MC synthesis was determined by the combination of the level of necessary enzymes and precursors and high EC. Nitrogen supplied MC synthesis with raw materials and precursors and offered the necessary enzymes during the series of complex processes, whereas phosphorus provided the necessary energy to support the series of complex synthesis processes and might also afford the necessary enzymes. These results provided a valuable perspective on the mechanism of nitrogen and phosphorus effects on MC synthesis. Our study can also contribute to the control of MC production by toxic cyanobacteria in eutrophicated water bodies.

\section{Acknowledgments}

This work was supported by the National Basic Research Program of China (Grant no. 2007CB407301) and the National Natural Science Foundation of China (50478115).

\section{References}

Atkinson, D.E., Walton, G.M., 1967. Adenosine triphosphate conservation in metabolic regulation. J. Biol. Chem. 242, 3239-3324.

Benzion, C., 1977. ATP in Lake Kinneret: indicator of microbial biomass or of phosphorus deficiency. Limnol. Oceanogr. 21, 231-236.

Bickel, H., Lyck, S., Utkilen, H., 2000. Energy state and toxin content-experiments on Microcystis aeruginosa (Chroococcales, Cyanophyta). Phycologia 39, 212-218.

Botes, D.P., Wessels, P.L., Kruger, H., Runnegar, M.T.C., Stntikarn, S., Sith, R.J., Barna, J.C.J., Willianms, D.H., 1985. Structural studies on cyanoginosins-LR, -YR, -YA and -YM, peptide toxins from Microcystis aeruginosa. J. Chem. Soc. Perkin Trans. 1, 2747-2748.

Boyer, G.L., Sullivan, J.J., Andersen, R.J., Harrison, P.J., Taylor, F.J.R., 1987. Effects of nutrient limitation on toxin production and composition in the marine dinoflagellate Protogonyaulax tamarenis. Mar. Biol. 96, 123-128.

Chapman, A.G., Fall, L., Atkinson, D.E., 1971. Adenylate energy charge in Escherichia coli during growth and starvation. J. Bacteriol. 108, 1072-1086.

Cheng, J.N., Mao, F.G., 1987. The Physiology of Microorganism. Fudan University Press, Shanghai, China, pp. 42-80.

Downing, T.G., Meyer, C., Gehringger, M.M., Van De Venter, M., 2005. Microcystin content of Microcystis aeruginosa is modulated by nitrogen uptake rate relative to specific growth rate or carbon fixation rate. Environ. Toxicol. 20, 257-262.

Editorial Board of Environment Protection Bureau of China (EPC), 2002. Monitoring and Determination Methods for Water and Wastewater, fourth ed. Environmental Science Press, Beijing, China.

Elke, D., Claudia, W., 2006. Cyanobacterial toxins-occurrence, biosynthesis and impact on human affairs. Mol. Nutr. Food Res. 50, 7-17.

Hegeman, R.H., 1969. Phosphorus metabolism in plants. Hortic. Science. 4, 311-314.

Hoeger, S.J., Schmid, D., Blom, J.F., Ernst, B., Dietrich, D.R., 2007. Analytical and functional characterization of microcystins $\left[\mathrm{Asp}^{3}\right] \mathrm{MC}-\mathrm{RR}$ and $\left[\mathrm{Asp}^{3}, \mathrm{Dhb}^{7}\right] \mathrm{MC}-\mathrm{RR}$ : consequences for risk assessment? Environ. Sci. Technol. 41, 2609-2616.

Jahnichen, S., Petaoldt, T., Benndorf, J., 2001. Evidence for control of microcystin dynamics in Bautzen Reservoir (Germany) by cyanobacterial population growth rates and dissolved inorganic carbon. Arch. Hydrobiol. 150, 177-196.

Kameyama, K., Sugiura, N., Inamori, Y., Maekawa, T., 2004. Characteristics of microcystin production in the cell cycle of Microcystis viridis. Environ. Toxicol. 19, 20-25.

Lawton, L.A., Edwards, C., Codd, G.A., 1994. Extraction and high-performance liquid chromatographic method for the 
determination of microcystins in raw and treated waters. Analyst 119, 1525-1530.

Lee, S.J., Jang, M.-H., Kim, H.-S., Yoon, B.-D., Oh, H.-M., 2000. Variation of microcystin content of Microcystis aeruginosa relative to medium $\mathrm{N}: \mathrm{P}$ ratio and growth stage. J. Appl. Microbiol. 89, 323-329.

Maria, V.A., Daniel, A.W., 2005. Effects of iron, ammonium and temperatures on microcystin content by a natural concentrated Microcystis aeruginosa population. Water Air Soil Pollut. 168, 235-248.

Moollan, R.W., Rae, B., Verbeek, A., 1996. Some comments on the determination of microcystin toxins in water by high-performance liquid chromatography. Analyst 121, 233-238.

Oh, H.-M., Lee, S.J., Jang, M.-H., Yoon, B.-D., 2000. Microcystin production by Microcystis aeruginosa in a phosphorous-limited chemostat. Appl. Environ. Microbiol. 66, 176-179.

Rinta-Kanto, J.M., Ouellette, A.J.A., Boyer, G.L., Twiss, M.R., Bridgeman, T.B., Wilhelm, S.W., 2005. Quantification of toxic Microcystis spp. during the 2003 and 2004 blooms in Western Lake Erie using quantitative real-time PCR. Environ. Sci. Technol. 39, 4198-4205.

Rippka, R., Deruelles, J., Waterbury, J.B., Herdman, M., Stanier, R.Y., 1979. Generic assignments, strain histories and properties of pure cultures of cyanobacteria. J. Gen. Microbiol. 111, 1-61.
Sakshaug, E., Holm-Hansen, O., 1977. Chemical composition of Skeletonema costatum (Grev.) Cleve and Pavlova (Monochrysis) lutheri (Droop) Green as a function of nitrate-, phosphate-, and iron-limited growth. J. Exp. Mar. Biol. Ecol. 29, 1-34.

Shi, H.X., Qu, J.H., Wang, A.M., Li, G.T., Lei, P.J., Liu, H.J., 2005. Primary investigation of blue-green algae and their toxins from water blooms in Guanting Reservoir of Beijing. Chem. J. Chin. Univ. 26, 1653-1655.

Song, L.R., Sano, T., Li, R.H., Watanabe, M., 1998. Microcystin production of Microcystis viridis (cyanobacteria) under different culture conditions. Phycol. Res. 46, 19-23.

Utkilen, H., Gjalme, N., 1992. Toxin production by Microcystis aeruginosa as a function of light in continuous cultures and its ecological significance. Appl. Environ. Microbiol. 58, 1321-1325.

Vezie, C., Rapala, J., Vaitomaa, J., Seitsonen, J., Sivonen, K., 2002. Effect of nitrogen and phosphorus on growth of toxic and nontoxic Microcystis strains and intracellular microcystin concentrations. Microbiol. Ecol. 43, 443-454.

Watanabe, M.F., Oishi, S., 1985. Effects of environmental factors on toxicity of cyanobacterium (Microcystis aeruginosa) under culture conditions. Appl. Environ. Microbiol. 42, 1342-1344.

Wicks, R.J., Thiel, P.G., 1990. Environmental factors affecting the production of peptide toxins in floating scums of the cyanobacterium Microcystis aeruginosa in a hypertrophic African reservoir. Environ. Sci. Technol. 24, 1413-1418. 